\title{
Solution of Newell - Whitehead - Segal Equation of Fractional Order by Using Sumudu Decomposition Method
}

\author{
Shams A. Ahmed ${ }^{1,2, *}$, Mohamed Elbadri ${ }^{1,2}$ \\ ${ }^{1}$ Department of Mathematics, Faculty of Sciences and Arts, Jouf University, Tubarjal, Saudi Arabia \\ ${ }^{2}$ Department of Mathematics, University of Gezira, Sudan
}

Received August 18, 2020; Revised September 29, 2020; Accepted October 19, 2020

\section{Cite This Paper in the following Citation Styles}

(a): [1] Shams A. Ahmed, Mohamed Elbadri, "Solution of Newell - Whitehead-Segal Equation of Fractional Order by Using Sumudu Decomposition Method," Mathematics and Statistics, Vol. 8, No. 6, pp. 631 - 636, 2020. DOI: $10.13189 / \mathrm{ms} .2020 .080602$.

(b): Shams A. Ahmed, Mohamed Elbadri (2020). Solution of Newell - Whitehead - Segal Equation of Fractional Order by Using Sumudu Decomposition Method. Mathematics and Statistics, 8(6), 631 - 636. DOI: 10.13189/ms.2020.080602.

Copyright $\mathrm{O} 2020$ by authors, all rights reserved. Authors agree that this article remains permanently open access under the terms of the Creative Commons Attribution License 4.0 International License

\begin{abstract}
Newell Whitehead Segal (NWS) equation has been used in describing many natural phenomena arising in fluid mechanics and hence acquired more attention. Studies in the past gave importance to obtaining numerical or analytical solutions of this kind of equations by employing methods like Modified Homotopy Analysis Transform method (MHATM), Adomian Decomposition method (ADM), Homotopy Analysis Sumudu Transform method (HASTM), Fractional Complex Transform (FCT) coupled with He's polynomials method (FCT-HPM) and Fractional Residual Power Series method (FRPSM). This research aims to demonstrate an efficient analytical method called the Sumudu Decomposition Method (SDM) for the study of analytical and numerical solutions of the NWS of fractional order. The coupling of Adomian Decomposition method with Sumudu transform method simplifies the calculation. From the numerical results obtained, it is evident that SDM is easy to execute and offers accurate results for the NWS equation than with other methods such as FCT-HPM and FRPSM. Therefore, it is easy to apply the coupling of Adomian Decomposition technique with Sumudu transform method, and when applied to nonlinear differential equations of fractional order, it yields accurate results.
\end{abstract}

Keywords Newell - Whitehead - Segal Equation, Sumudu Decomposition Method, Caputo Fractional Derivative

\section{Introduction}

Fractional calculus has played an important role in describing many dynamical phenomena in applied science and engineering fields. Dynamical phenomena are noticed in different type of scientific fields such as physics, chemistry, continuum mechanics [1], chaos theory [2], biotechnology [3], electrodynamics [4], and many other fields [5-7]. This feature of fractional calculus has appealed to many researchers in the past [8-12].

An NWS equation has been used in describing many natural phenomena arising in fluid mechanics and hence acquired more attention. This NWS equation describes the appearance of the stripe pattern in two-dimensional systems. Moreover, it has a lot of applications in fluid dynamics such as traveling wave patterns in binary fluids. Studies in the past gave importance to obtaining numerical or analytical solutions of this kind of equations by employing methods like Modified Homotopy Analysis Transform method (HASTM) [15], Adomian Decomposition method (ADM) [16], Homotopy Analysis Sumudu Transform method (HASTM) [17], Fractional Complex Transform (FCT) coupled with He's polynomials method (FCT-HPM) [18] and Fractional Residual Power Series method (FRPSM) [19]. 
As shown previously, in this study, we applied the Sumudu decomposition method (SDM) to find an analytical and numerical solution to NWS equations of fractional order. SDM, simple and directly without any restrictive assumption as usual, is going in other methods. In many research papers SDM has also been applied to solve intricate problems in engineering, mathematics and applied science [20-26].

In the present research, we consider the fractional model of Newell-Whitehead-Segal (NWS) equation in the operator form:

$$
\begin{gathered}
D_{\xi}^{v} \phi(x, \xi)=b \phi_{x x}(x, \xi)+k \phi(x, \xi)-\square \phi^{d}(x, \xi), \xi \geq \\
0,0<v \leq 1,
\end{gathered}
$$

with initial condition:

$$
\phi(x, 0)=g(x)
$$

where $D_{\xi}^{v} \phi(x, \xi)=\frac{\partial^{v} \phi(x, \xi)}{\partial \xi^{v}}, b, k$ and $h \in \mathfrak{R}$ such that $b, h>0$, and $d \in \square^{+}$. The term $\phi_{\xi}^{v}(x, \xi)$ represents the variation of $\phi(x, \xi)$ with respect to temporal variable $\xi$ at a set position, $\phi_{x x}(x, \xi)=\frac{\partial^{2} \phi}{\partial x^{2}}$ denotes the variation of $\phi(x, \xi)$ with spatial variable $x$ at a specific time and the remaining term $k \phi-h \phi^{d}$ signifies the effect of the source term.

\section{Preliminaries}

Definition 1: Let's Riemann-Liouville fractional integral operator [9] of order $v \geq 0$ for $\phi(\xi) \in \square_{\mu}, \mu \geq-1$, is:

$$
J^{v} \phi(\xi)=\left\{\begin{array}{l}
\frac{1}{\Gamma(v)} \int_{0}^{\xi}(\xi-\tau)^{\nu-1} \phi(\tau) d \tau, v>0, \xi>0, \\
\phi(\xi), \quad v=0 .
\end{array}\right.
$$

Definition 2: The Caputo fractional derivative operator of order [8] $v>0$ for $\phi(\xi) \in \square_{-1}^{k}, k \in \mathbb{N}$, is defined as:

$$
D_{\xi}^{v} \phi(\xi)=\frac{\partial^{v} \phi(\xi)}{\partial \xi^{v}}=\left\{\begin{array}{l}
J^{v-k} \phi^{k}(\xi), k-1<v \leq k, k \in \mathbb{N}, \\
\frac{\partial^{k} \phi(\xi)}{\partial \xi^{k}}, v=k, k \in \mathbb{N} .
\end{array}\right.
$$

Definition 3: The Sumudu transform [13] of the continuous function $\phi(\xi)$ is defined by:

$$
S[\phi(\xi)]=\frac{1}{\rho} \int_{0}^{\infty} \phi(\xi) e^{-\frac{\xi}{\rho}} d \xi=\phi(\rho) .
$$

Definition 4: The Sumudu transform of $\frac{\partial^{v} \phi(x, \xi)}{\partial \xi^{v}}$ w.r.t $\xi$ can be calculated as [14]:

$$
S\left[\frac{\partial^{v} \phi(x, \xi)}{\partial \xi^{v}}\right]=\rho^{-v} S[\phi(x, \xi)]-\sum_{k=0}^{r-1} \phi^{k}(x, 0) \rho^{-v+k}
$$

\section{Sumudu Decomposition Method (SDM)}

In this section, we will briefly discuss SDM, to solve fractional-order nonlinear (1.1). By applying the Sumudu transform (ST) to (1.1), we get

$$
\begin{gathered}
\rho^{-v} S[\phi(x, \xi)]-\rho^{-v} \phi(x, 0)=S\left[b \phi_{x x}(x, \xi)+\right. \\
\left.k \phi(x, \xi)-\square \phi^{d}(x, \xi)\right],
\end{gathered}
$$

substituting the (1.2) into (3.1), we obtain

$$
\begin{gathered}
S[\phi(x, \xi)]=g(x)+\rho^{v} S\left[b \phi_{x x}(x, \xi)+k \phi(x, \xi)-\right. \\
\left.\square \phi^{d}(x, \xi)\right] .
\end{gathered}
$$

On employing the inverse ST for (3.2), we get

$$
\begin{gathered}
\phi(x, \xi)=S^{-1}[g(x)]+S^{-1}\left[\rho ^ { v } S \left[b \phi_{x x}(x, \xi)+\right.\right. \\
\left.\left.k \phi(x, \xi)-\square \phi^{d}(x, \xi)\right]\right] .
\end{gathered}
$$

The SDM depends of the solution $\phi(x, \xi)$ in a series form as follows:

$$
\phi(x, \xi)=\sum_{m=0}^{\infty} \phi_{m}(x, \xi),
$$

the nonlinear term $\phi^{d}(x, \xi)$ is decomposed as follows:

$$
\phi^{d}(x, \xi)=\sum_{m=0}^{\infty} Q_{m},
$$

for some Adomian polynomials $Q_{m}$, it is defined as

$$
Q_{m}=\frac{1}{m !} \frac{d^{m}}{d \beta^{m}}\left[\left(\sum_{m=0}^{\infty} \beta^{m} \phi^{d}(x, \xi)\right)\right]_{\beta=0} \quad m=0,1,2, \ldots
$$

Substitution (3.4) and (3.5) to (3.3), we have

$$
\begin{array}{r}
S^{-1}[g(x)]+S^{-1}\left[\rho ^ { v } S \left[b\left(\sum_{m=0}^{\infty} \phi_{m}(x, \xi)\right)_{x x}+\right.\right. \\
\left.\left.k\left(\sum_{m=0}^{\infty} \phi_{m}(x, \xi)\right)-\square\left(\sum_{m=0}^{\infty} Q_{m}\right)\right]\right],
\end{array}
$$

Using (3.7), we define the following iterative formula [20]:

$$
\begin{gathered}
\phi_{0}(x, \xi)=S^{-1}[g(x)], \\
\phi_{k}(x, \xi)=S^{-1}\left[\rho ^ { v } S \left[b\left(\phi_{k-1}(x, \xi)\right)_{x x}+k\left(\phi_{k-1}(x, \xi)\right)-\right.\right. \\
\left.\left.\square\left(Q_{k-1}\right)\right]\right], k \geq 1 .
\end{gathered}
$$

Having determined these components, substitute it into $\phi(x, \xi)=\sum_{m=0}^{\infty} \phi_{m}(x, \xi)$, to obtain the solution in a series form.

\section{Elucidative Examples}

In this section, we demonstrate the applicability of the 
previous method by giving examples.

Example1. By substituting $b=1, k=-2, h=0$ in (1.1), to yield

$$
\phi_{\xi}^{v}(x, \xi)=\phi_{x x}(x, \xi)-2 \phi(x, \xi), \xi \geq 0,0<v \leq 1,
$$

with initial condition:

$$
\phi(x, 0)=e^{x},
$$

a time-fractional linear NWS equation.

By using SDM, (3.7) becomes

$$
\begin{array}{r}
\sum_{m=0}^{\infty} \phi_{m}(x, \xi)= \\
S^{-1}\left[e^{x}\right]+ \\
S^{-1}\left[\rho^{v} S\left[\left(\sum_{m=0}^{\infty} \phi_{m}(x, \xi)\right)_{x x}-2\left(\sum_{m=0}^{\infty} \phi_{m}(x, \xi)\right)\right]\right] .
\end{array}
$$

The iterative relation can be constructed from (4.3) given by:

$$
\begin{gathered}
\phi_{0}(x, \xi)=S^{-1}\left[e^{x}\right], \\
\phi_{k}(x, \xi)= \\
S^{-1}\left[\rho^{v} S\left[\left(\phi_{k-1}(x, \xi)\right)_{x x}-2\left(\phi_{k-1}(x, \xi)\right)\right]\right], k \geq 1 .
\end{gathered}
$$

Therefore, using SDM, the first few iterative solutions are

$$
\begin{aligned}
& \phi_{0}(x, \xi)=e^{x}, \\
& \phi_{1}(x, \xi)=-\frac{e^{x} \xi^{v}}{\Gamma[1+v]}, \\
& \phi_{2}(x, \xi)=\frac{e^{x} \xi^{2 v}}{\Gamma[1+2 v]}, \\
& \phi_{3}(x, \xi)=-\frac{e^{x} \xi^{3 v}}{\Gamma[1+3 v]}, \\
& \phi_{4}(x, \xi)=\frac{e^{x} \xi^{4 v}}{\Gamma[1+4 v]} .
\end{aligned}
$$

So, the solution $\phi(x, \xi)$ in series form is given by;

$$
\phi(x, \xi)=e^{x}\left[1-\frac{\xi^{v}}{\Gamma[1+v]}+\frac{\xi^{2 v}}{\Gamma[1+2 v]}-\frac{\xi^{3 v}}{\Gamma[1+3 v]}+\frac{\xi^{4 v}}{\Gamma[1+4 v]}\right],
$$

Putting $v=1$, we get

$$
\phi(x, \xi)=e^{x-\xi} .
$$

Fig. 1(a) and Fig. 1(b) respectively show the exact solution and approximate solutions yielded by the SDM at $v=1$. Furthermore, the analysis of absolute errors is summarized in Table 1.

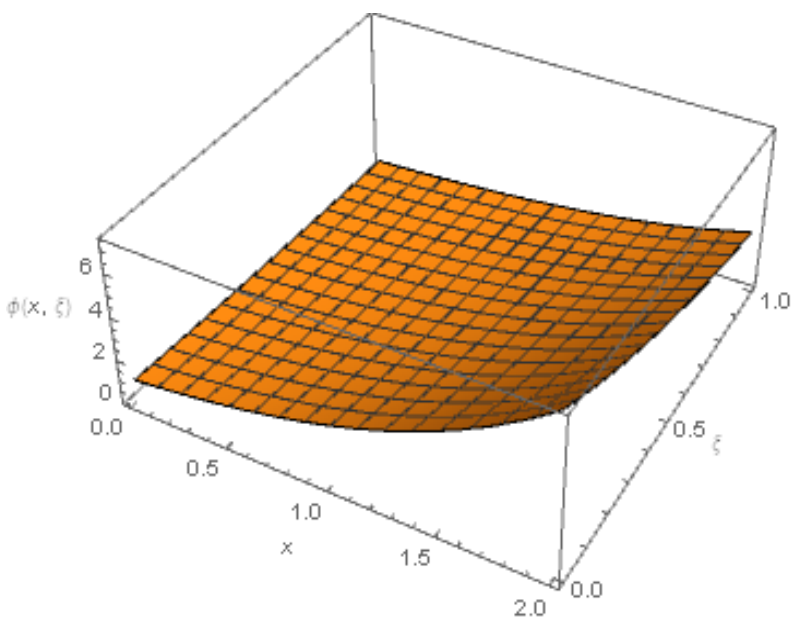

Figure 1(a). Exact solution

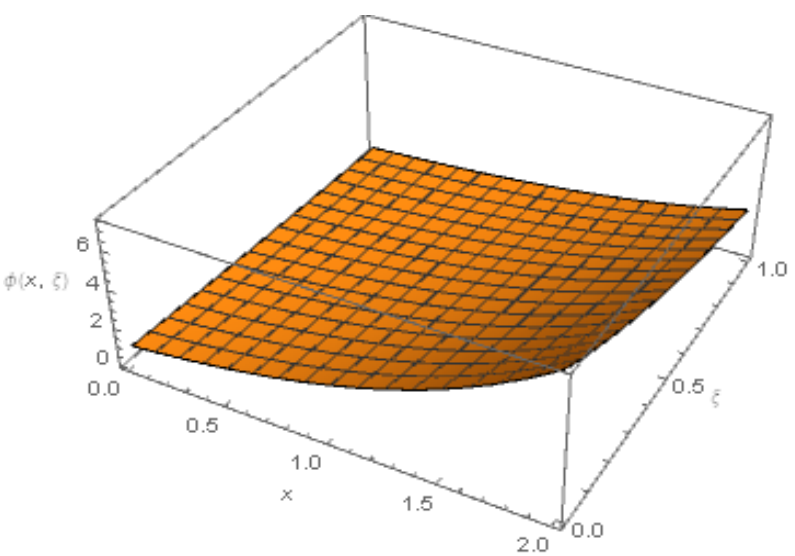

Figure 1(b). SDM

Example 2. By substituting $b=1, k=2, h=-3$ and $d=2$ in (1.1),

$$
\begin{gathered}
\phi_{\xi}^{v}(x, \xi)=\phi_{x x}(x, \xi)+2 \phi(x, \xi)-3 \phi^{2}(x, \xi), \xi \geq 0,0< \\
v \leq 1,
\end{gathered}
$$

with initial condition:

$$
\phi(x, 0)=\gamma,
$$

a time-fractional non- linear NWS equation.

Using the previous aforesaid in Example 1, we get: 


$$
\begin{aligned}
& \phi_{0}(x, \xi)=\gamma \\
& \phi_{1}(x, \xi)=\frac{2 \xi^{v} \gamma}{\Gamma[1+v]}-\frac{3 \xi^{v} \gamma^{2}}{\Gamma[1+v]}, \\
& \phi_{2}(x, \xi)=\frac{4 \xi^{2 v} \gamma}{\Gamma[1+2 v]}-\frac{18 \xi^{2 v} \gamma^{2}}{\Gamma[1+2 v]}+\frac{18 \xi^{3 v} \gamma^{3}}{\Gamma[1+3 v]}, \\
& \phi_{3}(x, \xi)=\frac{8 \xi^{3 v} \gamma}{\Gamma[1+3 v]}-\frac{60 \xi^{3 v} \gamma^{2}}{\Gamma[1+3 v]}+\frac{144 \xi^{3 v} \gamma^{3}}{\Gamma[1+3 v]}-\frac{108 \xi^{3 v} \gamma^{4}}{\Gamma[1+4 v]}- \\
& \frac{12 \xi^{3 v} \gamma^{2} \Gamma[1+2 v]}{\Gamma[1+v]^{2} \Gamma[1+3 v]}+\frac{36 \xi^{3 v} \gamma^{3} \Gamma[1+2 v]}{\Gamma[1+v]^{2} \Gamma[1+3 v]}-\frac{27 \xi^{3 v} \gamma^{4} \Gamma[1+2 v]}{\Gamma[1+v]^{2} \Gamma[1+3 v]}, \\
& \phi_{4}(x, \xi)=\frac{16 \xi^{4 v} \gamma}{\Gamma[1+4 v]}-\frac{168 \xi^{4 v} \gamma^{2}}{\Gamma[1+4 v]}+\frac{648 \xi^{4 v} \gamma^{3}}{\Gamma[1+4 v]}-\frac{1080 \xi^{4 v} \gamma^{4}}{\Gamma[1+4 v]}+ \\
& \frac{648 \xi^{4 v} \gamma^{5}}{\Gamma[1+4 v]}-\frac{24 \xi^{4 v} \gamma^{2} \Gamma[1+2 v]}{\Gamma[1+v]^{2} \Gamma[1+4 v]}+\frac{144 \xi^{4 v} \gamma^{3} \Gamma[1+2 v]}{\Gamma[1+v]^{2} \Gamma[1+4 v]}- \\
& \frac{270 \xi^{4 v} \gamma^{4} \Gamma[1+2 v]}{\Gamma[1+v]^{2} \Gamma[1+4 v]}+\frac{162 \xi^{4 v} \gamma^{5} \Gamma[1+2 v]}{\Gamma[1+v]^{2} \Gamma[1+4 v]}-\frac{48 \xi^{4 v} \gamma^{2} \Gamma[1+3 v]}{\Gamma[1+v] \Gamma[1+2 v] \Gamma[1+4 v]} \\
& +\frac{288 \xi^{4 v} \gamma^{3} \Gamma[1+3 v]}{\Gamma[1+v] \Gamma[1+2 v] \Gamma[1+4 v]}-\frac{540 \xi^{4 v} \gamma^{4} \Gamma[1+3 v]}{\Gamma[1+v] \Gamma[1+2 v] \Gamma[1+4 v]}+\frac{324 \xi^{4 v} \gamma^{5} \Gamma[1+3 v]}{\Gamma[1+v] \Gamma[1+2 v] \Gamma[1+4 v]}
\end{aligned}
$$

Therefore, the solution $\phi(x, \xi)$ in series form is given by;

\begin{tabular}{|c|c|c|c|c|c|}
\hline \multirow{2}{*}{$\xi$} & \multirow[b]{2}{*}{ Exact } & \multicolumn{2}{|c|}{ SDM } & \multicolumn{2}{|c|}{ FCT-HPM [18] } \\
\hline & & Fifth Appr. Sol & Absolute Error & Fifth Appr. Sol & Absolute Error \\
\hline $\begin{array}{l}0.16 \\
0.32 \\
0.48 \\
0.64 \\
0.80 \\
0.96\end{array}$ & $\begin{array}{c}0.00137635 \\
0.00189393 \\
0.0026054 \\
0.00358269 \\
0.00492384 \\
0.00676192\end{array}$ & $\begin{array}{c}0.00137632 \\
0.00189299 \\
0.0025978 \\
0.00354881 \\
0.00481432 \\
0.00677288\end{array}$ & $\begin{array}{l}2.80827 \\
\times 10^{-8} 9.47645 \\
\times 10^{-7} \\
7.60049 \times 10^{-6} \\
3.38792 \times 10^{-5} \\
1.0952 \times 10^{-4} \\
2.89035 \times 10^{-4}\end{array}$ & $\begin{array}{l}0.00137393 \\
0.00187387 \\
0.00253329 \\
0.00339589 \\
0.00451565 \\
0.00595679\end{array}$ & $\begin{array}{l}2.41742 \\
\times 10^{-6} 2.00623 \times 10^{-5} \\
7.21125 \times 10^{-5} \\
1.86797 \times 10^{-4} \\
4.08187 \times 10^{-4} \\
8.05131 \times 10^{-4}\end{array}$ \\
\hline
\end{tabular}

$$
\phi(x, \xi)=\phi_{0}(x, \xi)+\phi_{1}(x, \xi)+\phi_{2}(x, \xi)+\phi_{3}(x, \xi)+\phi_{4}(x, \xi)+\ldots
$$

putting $v=1$, we get

$$
\phi(x, \xi)=\frac{-\frac{2}{3} \gamma e^{2 \xi}}{-\frac{2}{3}+\gamma-\gamma e^{2 \xi}} .
$$

Table 1. Comparison between Exact solution and Approximate solution of Example 1 at $v=1$.

\begin{tabular}{|c|c|c|c|c|}
\hline$x$ & $\xi$ & Exact & Approximation & Absolute Error \\
\hline 0.2 & 0.16 & 6.296538261026657 & 6.296544549363063 & $6.28834 \times 10^{-6}$ \\
& 0.32 & 5.365555971121974 & 5.365752049033128 & $1.96078 \times 10^{-4}$ \\
& 0.48 & 4.572225195142159 & 4.573676761867118 & $1.45157 \times 10^{-3}$ \\
& 0.64 & 3.896193301795214 & 3.90215934335963 & $5.96604 \times 10^{-3}$ \\
& 0.80 & 3.320116922736547 & 3.33788294175694 & $1.7766 \times 10^{-2}$ \\
& 0.96 & 2.82921701435156 & 2.872373195690292 & $4.31562 \times 10^{-2}$ \\
\hline
\end{tabular}

Table 2. Comparison of results between SDM and FCT-HPM, at $\gamma=0.001$, and $v=1$ for Example 2 
Table 3. Comparison of results between SDM and FRPSM, at $\gamma=0.001$, and $v=1$ for Example 2

\begin{tabular}{|c|c|c|c|c|c|}
\hline \multirow{2}{*}{$\xi$} & \multirow{2}{*}{ Exact } & \multicolumn{2}{|c|}{ SDM } & \multicolumn{2}{c|}{ FRPSM [19] } \\
\cline { 3 - 6 } & Fifth Appr. Sol & Absolute Error & Fifth Appr. Sol & Absolute Error \\
\hline 0.16 & 0.00137635 & 0.00137632 & 2.80827 & & \\
0.32 & 0.00189393 & 0.00189299 & $\times 10^{-8} 9.47645$ & 0.00137629 & $6.07525 \times 10^{-8} 1.209$ \\
0.48 & 0.0026054 & 0.0025978 & $\times 10^{-7}$ & 0.00189273 & $\times 10^{-6}$ \\
0.64 & 0.00358269 & 0.00354881 & $7.60049 \times 10^{-6}$ & 0.00259692 & $8.48258 \times 10^{-6}$ \\
0.80 & 0.00492384 & 0.00481432 & $3.38792 \times 10^{-5}$ & 0.00354672 & $3.59701 \times 10^{-5}$ \\
0.96 & 0.00676192 & 0.00677288 & $1.0952 \times 10^{-4}$ & 0.00481023 & $1.13604 \times 10^{-4}$ \\
& & & $2.89035 \times 10^{-4}$ & 0.00646583 & $2.96092 \times 10^{-4}$ \\
\hline
\end{tabular}

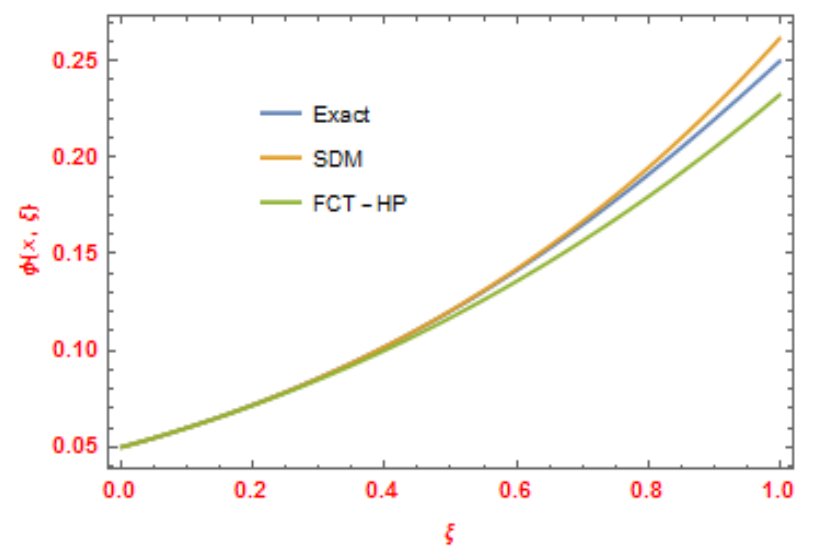

Figure 2. Comparison between exact solution, SDM and FCT- HPM at $\gamma=0.001$, and $v=1$ for Example 2

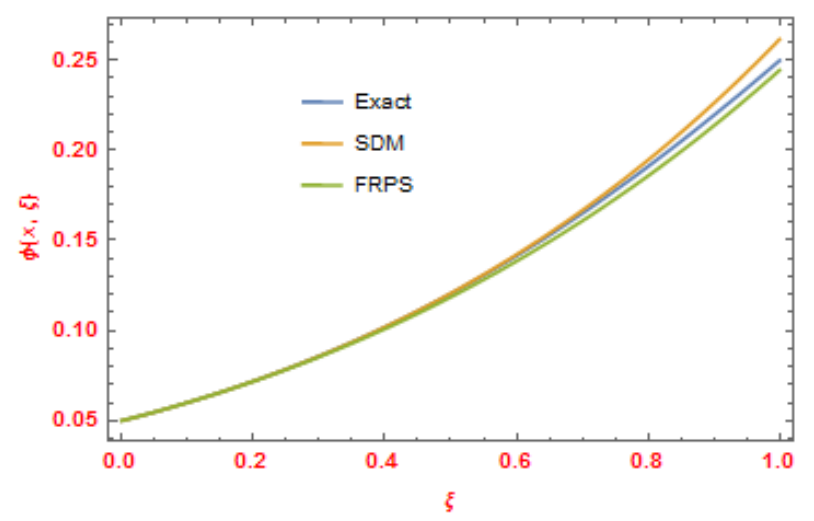

Figure 3. Comparison between exact solution, SDM and FRPSM at $\gamma=0.001$, and $v=1$ for Example 2

The numerical results shown in Tables 2 and 3 and Figs. 2 and 3 illustrate that SDM offers accurate results in comparison with FCT-HPM [18] and FRPSM [19].

\section{Conclusions}

In this paper, SDM had been successfully applied to get approximate solutions of Newell-Whitehead-Segal (NWS) equation of fractional order. It is clearly seen from the numerical results that SDM is easy to execute and offers accurate results for the NWS equation. Hence, SDM is a simple and effective method to obtain approximate and analytical solutions for many differential fractional equations.

\section{REFERENCES}

[1] C.S. Drapaca, S. Sivaloganathan, A fractional model of continuum mechanics, Journal of Elasticity, vol. 107,105-123, 2012.

[2] D. Baleanu, G.C. Wu, S.D. Zeng, Chaos analysis and asymptotic stability of generalized Caputo fractional differential equations, Chaos solitons fractals, vol. 102, 99-105, 2017.

[3] D. Kumar, A.R. Seadwy, A.K. Joarder, Modified kudryashov method via new exact solutions for some conformable fractional differential equations arising in mathematical biology, Chines journal of physics, vol. 56, no. 1, 75-85, 2018.

[4] H. Nasrolahpour, A note on fractional electrodynamics, Communications in Nonlinear Science and Numerical Simulation, vol. 18, no. 9, 2589-2593, 2013.

[5] D.G. Prakasha, P. Veeresha, H.M. Baskonus, Residual power series method for fractional Swift - Hohenberg equation. Fractal and fractional, vol. 3, no. 1, 1-16, 2019.

[6] P. Agarwal, A.A. El-sayed, non - standard finite difference and Chebyshev collocation methods for solving fractional diffusion equation, Phys, vol. 500, 40-49, 2018.

[7] D.G. Prakasha, P. Veeresha, M.S Rawashdeh, Numerical solution for $(2+1)$ dimensional time - fractional coupled Burger equation using fractional natural decomposition method, Mathematical Methods in the Applied Sciences, vol.42, no.1, 1-19,2019.

[8] M. Caputo, Elasticita e Dissipazione, Bologna, Zanichelli, 1969.

[9] K.S. Miller, B. Ross, An introduction to fractional calculus and fractional differential equations, Wiley, New York, 1993.

[10] I. Podlubny, Fractional differential equations. Academic Press, New York, 1999.

[11] A. Repaci, Nonlinear Dynamical Systems: On the Accuracy of Adomian's Decomposition Method, Appl. Math. Lett, 
vol.3, no. 3, 35-39, 1990 .

[12] S.J. Liao, Homotopy analysis method: a new analytic method for nonlinear problems, Appl. Math. Mech, vol. $19,957-962,1998$

[13] F.B.M. Belgacem, A.A. Karaballi, Sumudu Transform Fundamental Properties Investigations and Application, Journal of Applied Mathematics and Stochastic Analysis, 3,103-118, 2003.

[14] V.B.L. Chaurasia, J. Singh, Application of Sumudu transform in Schrodinger equation occurring in quantum mechanics, Applied Mathematical Sciences, 4(7),28432850,2010

[15] S. Kumar, A. Kumar, S. Momani, M. Aldhaifallah, K. S. Nisar, Numerical solutions of nonlinear fractional model arising in the appearance of the strip patterns in two-dimensional systems, Advances in Difference Equations, 2019. https://doi.org/10.1186/s13662-019-2334-

[16] A. Prakash, V. Verma, Numerical method for fractional model of Newell-Whitehead-Segel equation, Front. Phys, 2019. https://doi.org/10.3389/fphy.2019.00015

[17] D. Kumar; R. Prakash, Numerical approximation of Newell Whitehead-Segel equation of fractional order, Nonlin Eng,5,81-86, 2016.

[18] S.O. Edeki, O.P. Ogundile, B. Osoba, G.A. Adeyemi, F.O. Egara, A.S. Ejoh, Coupled FCT-HP for Analytical Solutions of the Generalized Time-fractional Newell-Whitehead-Segel Equation, WSEAS Trans. Syst. Control, 13,266-274, 2018.

[19] R. Saadeh, M. Alaroud, M. Al-Smadi, R.R. Ahmad, U. Khair,
Application of Fractional Residual Power Series Algorithm to Solve Newell-Whitehead-Segel Equation of Fractional Order, Symmetry 11(12),1-13,2019.

[20] S. Ahmed and T. Elzaki, On the comparative study integro-differential equations using difference numerical methods, Journal of King Saud University-Science, vol. 32, no. 1, pp. 84-89, 2020.

[21] S. Ahmed and M. Elbadri, M. Z. Mohamed, A New Efficient Method for Solving Two-Dimensional Nonlinear System of Burger's Differential Equation, Journal Abstract and Applied Analysis, vol. 2020, pp. 1-7.

[22] S. Ahmed, A comparison between modified Sumudu decomposition method and homotopy perturbation method, Applied Mathematics, vol. 9, no. 3, pp. 199-206, 2018.

[23] S. Ahmed and T. Elzaki, Solution of heat and wave-like equations by adomian decomposition Sumudu transform method, British Journal of Mathematics \& Computer Science, vol. 8, no. 2, pp. 101-111, 2015.

[24] S. Ahmed and T. Elzaki, A comparative study of Sumudu decomposition method and Sumudu projected differential transform method, World Applied Sciences Journal, vol. 31, no. 10, pp. 1704-1709, 2014.

[25] S. Ahmed, Application of Sumudu Decomposition Method for Solving Burger's Equation, Advances in Theoretical and Applied Mathematics, vol.9;(2014), 23-26.

[26] S. Ahmed and T. Elzaki, the solution of nonlinear Volterra integro-differential equations of second kind by combine Sumudu transforms and Adomian decomposition method, International Journal of Advanced and Innovative Research, vol. 2, no. 12, pp. 90-93, 2013. 